\title{
MANEJO DEL ENFRIAMIENTO LIQUIDO EN ORDENADORES
}

AUTORES: Jennifer Elizabeth Chóez Calle ${ }^{1}$

Carlos Andrés Villacreses Parrales ${ }^{2}$

José E. Álava Cruzatty ${ }^{3}$

\section{DIRECCIÓN PARA CORRESPONDENCIA: (jenniferelizachoezcalle@gmail.com)}

Fecha de recepción: 05/03/2019

Fecha de aceptación: 10/04/2019

\section{RESUMEN}

El presente trabajo de investigación se fundamentó en dar a conocer como beneficia o afecta la implementación del enfriamiento líquido en ordenadores en la actualidad; dando a entender que al hacer el uso correcto de los componentes necesarios para su adquisición, e implementar esta técnica, los usuarios no cometan errores y puedan así afectar al ordenador. En la investigación presente se utilizó diferentes métodos el cual predomino el método análisissíntesis. Esta técnica se basa en la utilización del agua, la cual se transporta mediante tubos los cuales estarán conectados a los diferentes componentes del case para poder lograr disipar la acumulación del calor dentro del mismo, cosa que puede hacer el proceso que habitualmente traen incorporados los ordenadores; por lo tanto no es una técnica la cual permita bajar el total de temperatura presente en cada uno sus componentes. Se encuentra fabricado para las nuevas actualizaciones de case, permitiendo una disminución de la temperatura interna de sus principales componentes; tal que prácticamente el ordenador puede realizar diferentes tareas sin que sufra un mínimo desgaste en su sistema; por lo tanto es prioritario mantener el equipo bien refrigerado. Con el avanzar del tiempo las tecnologías emergentes integradas en un ordenador necesitarán un enfriamiento muy eficiente y eficaz, el cual llene todas las expectativas de mantener en un buen estado y funcionamiento del 99,9\% los mismos para que así los usuarios no tengan presentes inconvenientes al querer usar sus ordenadores y su funcionamiento sea óptimo.

1 Estudiante de la Carrera Tecnologías de la Información. Bachillerato. Estudiante de la Universidad Estatal del Sur de Manabí. Jipijapa, Manabí y Ecuador. jenniferelizachoezcalle@gmail.com

2 Docente de la Carrera Tecnologías de la Información. Bachillerato. Docente de la Universidad Estatal del Sur de Manabí. Jipijapa, Manabí y Ecuador. carlosvillacresesparrales23@gmail.com

3 Estudiante de la Carrera Tecnologías de la Información. Bachillerato. Estudiante de la Universidad Estatal del Sur de Manabí. Jipijapa, Manabí y Ecuador. carlosvillacresesparrales23@gmail.com 
PALABRAS CLAVE:Enfriamiento liquido; ordenador; sistema; temperatura.

\title{
USE OF LIQUID COOLING IN COMPUTERS
}

\begin{abstract}
The present research work was based on making known how it benefits or affects the implementation of liquid cooling in computers today; implying that by making the correct use of the components necessary for its acquisition, and implementing this technique, users do not make mistakes and can thus affect the computer. Scientific research methods were used, predominantly the analysis-synthesis method. This technique is based on the use of water, which is transported by tubes which will be connected to the different components of the case to be able to dissipate the accumulation of heat inside it, which can be done by the process that computers usually incorporate ; therefore it is not a technique which allows to lower the total temperature present in each one of its components. It is manufactured for new case updates, allowing a decrease in the internal temperature of its main components; such that practically the computer can perform different tasks without suffering minimal wear and tear on your system; therefore it is a priority to keep the equipment well refrigerated. With the advance of time the emerging technologies integrated into a computer will need a very efficient and efficient cooling, which fulfills all the expectations of maintaining in a good state and functioning of $99.9 \%$ the same so that users do not have any inconveniences in mind When you want to use your computers and their operation is optimal.
\end{abstract}

Keywords:computer;Liquid cooling; system; temperature.

\section{INTRODUCCIÓN}

El enfriamiento líquido, sistema de refrigeración o en inglés “watercooling”, es una técnica que se implementan en los actualesordenadores para controlar la temperatura de los componentes, en la cual se utiliza agua en vez de ventiladores o disipadores de calor(dentro del Case) así se obtienen excelentes resultados como mejorar el rendimiento y poder bajar la temperatura existente en el ordenador. 
Se pretende que los usuarios adquieran e implementen esta técnica para así mejorar el rendimiento del ordenador puesto que es recomendable para aquellos que hacen uso de ciertas actividades que requieren que el mismo se esfuerce mucho más, lo cual permite llevar su capacidad de procesamiento al máximo sin que ocurra un sobrecalentamiento.

Un ordenador llega al límite de su rendimiento debido a sus aplicaciones que se están ejecutando o en cuales se está trabajando ocupen el mayor espacio tanto en memoria como en procesador, el mismo sufre de un aumento considerable de la temperatura es ahí necesario que intervenga una refrigeración;En mucho de los casoses necesario el aislamiento adecuado de los componentes del case antes que se realice este tipo de técnica, que advierte las fallas que se pueden presentar en los mismos.

Dentro de las técnicas de enfriamiento liquido se dan a conocer el "Custom” y "All in One” la cual se diferencian de que la primera hace uso de tubos y requiere de un mayor mantenimiento; y la segundaestá compuesta de bomba y radiador lo cualno requiere mantenimiento por lo que se mejora el rendimiento.

El utilizar ventiladores en los equipos favorece a las aplicaciones, pero el enfriamiento líquido contribuye para que se ejecuten de una manera rápida y no se presenten inconvenientes al trabajar con las aplicaciones.

Tener un ordenador no solamente implica que se mantenga bienrefrigerado para mejorar su rendimiento sino también para alargar el ciclo de vida de sus componentesy así tengan una mayor durabilidad; por esta razón es importante tener conocimiento de las técnicas que se van implementando en la actualidad.

Se debe considerar que para implementar este tipo de enfriamiento se debe hacer un análisis de los factores que se relacionan con el ordenador, como por ejemplo: suscircuitos.

Esta técnica en la actualidad no solo se ha implementado en los ordenadores con tecnología avanzada; sino también en los Smartphone usando un esquema muy similar pero mucho más reducido como son las tuberías de calor o mayormente conocidos como "HeatPipe”, es una sustancia liquida que recorre las zonas en las que se produce el alza de temperatura (calor),y su principal función es bajar o reducir dicho calor existente en el mismo.

Cabe recalcar que esta técnica se implementa en las computadoras actuales puesto que las antiguas versiones no funcionaran con igual eficiencia, su funcionamiento se basa en que a 
través de tubos el agua pasa por todos los componentes tanto así que saca el calor que se encuentre en el mismo volviéndose en un ciclo repetitivo.

El objetivo, es brindar a los lectores una investigación relacionada con los sistemas de enfriamiento líquido con más frecuencia usados ya sea para mantener el ordenador optimo así como mejorar el rendimiento; es decir de cualquier manera alargar el tiempo de vida del procesador y sus componentes.

El impacto que tendrá en los usuarios es que aceptable ya que es una técnica muy eficazeficiente y además muy innovadora que permitirá el buen funcionamiento de un ordenador siempre que se haga uso de una técnica apropiada.

\section{DESARROLLO}

La refrigeración líquida o sistema de refrigeración, basa su ejecución en las propiedades térmicas de los líquidos que son conductivos; necesitade un componente como lo es la bomba la cual su función principal consistirá en transportar el agua por todos los componentes que contiene el ordenador; cuyo líquido que se transporta por todos los circuitos pasa por la memoria RAM, procesador, placa base y la tarjeta gráfica, como se muestra en el Gráfico N.1; una vez que el líquido enfría los componentes este se calienta, al mismo tiempo el líquido pasa por el radiador y los ventiladores internos del mismo hacen que el líquido se enfríe y vuelva a realizar el mismo proceso.(Rodrigo , 2015).

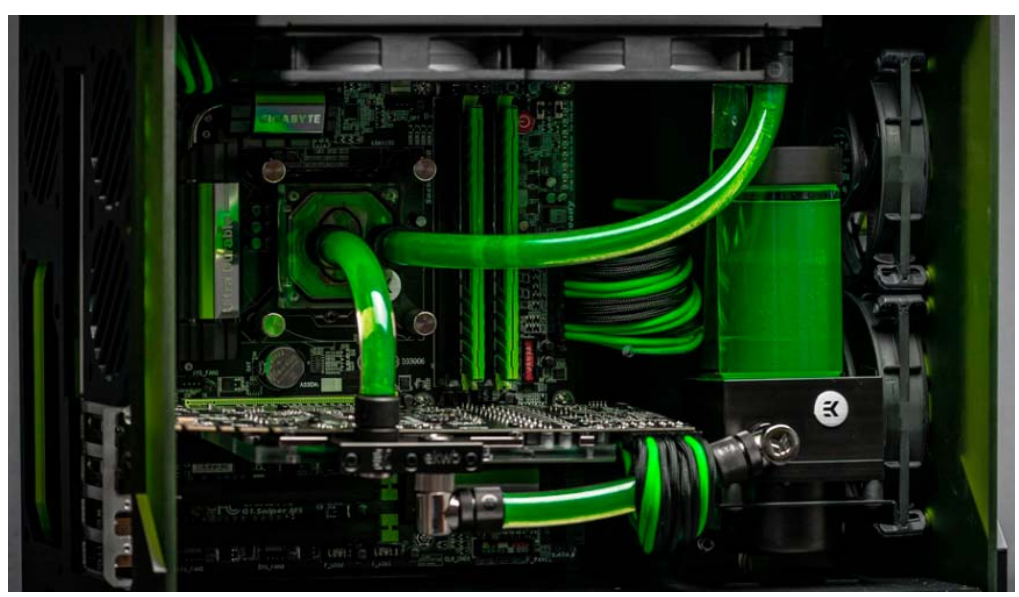

GRÁFICO N. 1:Refrigeración líquida de un ordenador

\section{Enfriamiento Liquido VS. Enfriamiento tradicional (aire)}


Como es de saber cuándo una persona compra un ordenador ya armado, esto no genera preocupación por lo que ya viene con un enfriamiento adecuado para el ordenador; pero para aquellos que desean armar uno, se recomienda conocer el tipo de enfriamiento adecuado para los componentes internos del ordenador según la utilización que se la va a dar al mismo.

Con el pasar de los años se ha venido utilizando el enfriamiento tradicional, lo cual en muchos de los casos no es apto para aquellas personas que desean llevar al máximo el ordenador y a cada uno de sus componentes como es el procesador, la RAM, tarjeta gráfica y en alguno más en función al modelo.

Y es por esta razón saber la diferencia entre el sistema de enfriamiento tradicional y el enfriamiento líquido, lo cual se detalla a continuación:

\section{Enfriamiento con aire o tradicional}

El enfriamiento se ha implementado en mundo de la tecnología, haciendo uso de ventiladores; lo cual expulsa el calor existente en los componentes del ordenador.

Este tipo de enfriamiento tiene sus PROS como son:

$\checkmark$ Son muy económicos.

$\checkmark$ Son muy fáciles de colocar, lo cual solo se debe ajustar tornillos y saber su ubicación correcta.

$\checkmark$ Solo se debe limpiar el polvo regularmente.

Entre sus CONTRAS se menciona:

$\checkmark$ Son muy ruidos especialmente si la computadora está a su límite de uso.

$\checkmark$ Si se necesita utilizar más ventiladores también se necesitar mayor espacio; es decir un case más grande.

$\checkmark$ Tal es el caso que son menos eficientes, puesto que enfría todo a la vez mas no uno específico.

\section{Sistemas de enfriamiento líquido}

En la actualidad existen diferentes enfriamientos líquidos los cuales podemos mencionar: 
Sistemas por inmersión:Su principal función es sumergir el ordenador que se va a enfriar en un el líquido que tenga poca conductividad eléctrica, esto puede ser aceite o agua.

$\checkmark$ Sistemas de refrigeración con metal líquido: Este sistema usa una bomba con tubos por el que circula el líquido, el cual circula por todos los componentes.

$\checkmark$ Sistema de refrigeración Termoeléctrica: Este sistema hace uso de técnicas denominadas industriales para expulsar el calor que se encuentra en los componentes de un ordenador.

$\checkmark$ Sistemas de refrigeración Heatpipes: Esta es una técnica de flujo continuo natural que dilata los líquidos; al calentarse circulan para que realicen su respectivo enfriamiento y así con ello enfriar al procesador.

$\checkmark$ Sistemas de refrigeración de cambio de fase: Este refrigerante hace uso de gases que sean ideales y termodinámicos que funcionan para crear máquinas capaces de hacer que los circuitos del ordenador se enfríen.

$\checkmark$ Sistemas de refrigeración por criogenia: Este sistema se basa el utilizar hielo seco o nitrógeno líquido, lo cual requiere mantener el ordenador a una temperatura baja manteniendo así los componentes bien fríos.

$\checkmark$ Sistemas de refrigeración por propulsión de aire electroestático: Esta técnica es experimental ya que hace uso de una bobina de viento y esta permite que se realice el enfriamiento proporcionalmente en los componentes que tiene el ordenador.

\section{Enfriamiento líquido}

Este tipo de enfriamiento utiliza un líquido, normalmente agua, como sustancia para enfriar cada uno de los elementos del ordenador. La particularidad de esta técnica es que expulsa el calor de los diferentes elementos, muy parecido a los reactores nucleares; así permitiendo que su temperatura disminuya.

Su principal función es expulsar el calor de cada uno de los elementos del Case, lo que no se puede hacer con elque viene incorporado en los ordenadores por defecto (aire o tradicional);esta técnica es mucho más complicado de armar ya que se requiere bloques de agua, un radiador, tubos y bombas. Por lo que un usuario común no optaría por este métodoya que resulta costoso y complicado de implementar, este método, seria mayormente 
implementado por usuarios Gamers o aquellos que realizan actividades exigentes.(Franco , 2013).

\section{Elementos necesarios para implementar la refrigeración líquida}

Para implementar una refrigeración líquida "parte por parte” o por "kits”; es recomendable la primera, por lo que algunos kits no satisfacen a los “overclocking” y “modders”. La marca en algunos de los kits es muy imprescindibles, puesto que el diseño de sus componentes mejore el rendimiento que se requiere para optimizar el ordenador.

$\checkmark$ Bloque del microprocesador:Su función es intercambiala temperatura (calor) con el procesador, para así aumentar el rendimiento en su reloj como en el voltaje.

$\checkmark$ Bloque para disco duro:permite que el calor se intercambie entre los discos duros existentes, elemento que produce mucho calor con su uso a través del tiempo, por lo que muchos de los casos pueden causar fallas de escritura e incluso de lectura, incluido la pérdida de sus datos.

$\checkmark$ Bloque para tarjeta gráfica:la función principal de este bloque es intercambiar el calor que se presenta en el circuito de la misma.

$\checkmark$ Pasta térmica:elemento utilizado para la conducción del calor generado por los dispositivos del ordenador, como el procesador y sus diferentes circuitos.

$\checkmark$ Bomba:su función principal es conducir el agua de los circuitos para así que esta no se quede estático dentro del ordenador.

$\checkmark$ Depósito:unidad que permite sufragar las pérdidas a través de las reservas de fluidos; e incluso cumple las funciones de los refrigerantes.

$\checkmark$ Radiador:Por este componente pasa el líquido y se enfría para que así vuelva realizar su ciclo repetitivo. (Morza, 2018).

\section{Ventajas:}

$\checkmark$ Su rendimiento es notoriamente más avanzado a los disipadores por aire.

$\checkmark$ Se puede refrigerar todos los elementos de un ordenador.

$\checkmark$ No es ruidoso al momento que se está ejecutando, aunque depende de la bomba y los ventiladores que se obtenga.

\section{Desventajas:}

$\checkmark$ Su adquisición podría resultar costosa. 
Se pueden presentar inconveniente al momento de armarlos o instalarlos.

$\checkmark$ Exigeuna constante revisión o mantenimiento.

$\checkmark$ Es necesario rellenar continuamente el líquido refrigerante.

$\checkmark$ En caso de que se dañe uno de los tubos o exista una polilla en los mismos podría dañar el ordenador.

$\checkmark$ Requiere Case compatibles para realizar la refrigeración líquida.

\section{Materiales a emplear en una refrigeración liquida}

El realizar una refrigeración requiere de técnicos expertos que conozcan el cómo funciona su implementación y sobre todo de sus beneficios en un ordenador; entre sus componentes encontramos los siguientes:

$\checkmark$ El radiador.

$\checkmark$ El tubing (se trata del tubo por donde circula el refrigerante).

$\checkmark$ El refrigerante (glicol de etileno o agua destilada).

$\checkmark$ Accesorios para el montaje.

$\checkmark$ La bomba (la cual produce que el refrigerante circule y cumpla su ciclo).

$\checkmark$ El deposito (Lugar donde el refrigerante regresara cada vez que cumpla su ciclo).

$\checkmark$ Bloque del CPU (Case compatible para la implementación de este técnica).

$\checkmark$ Kits (las instrucciones e indicaciones para la debida instalación).

Al finalizar la debida instalación de esta técnica se debe realizar un testeo a la refrigeración, la cual es similar a la de un disipador, omitiendo el detalle de que al usar agua, la temperatura tarda más en cargar puesto que debe de calentarse todo el circuitode igual manera que la de enfriarse todos sus componentes.

\section{Tipos de Refrigeración Liquida}

\section{Refrigeración líquida “Custom”}

Este tipo de refrigeración se implementaparte por parte. Tubos, racores, bomba; para ello se necesita mucha experiencia para evitar fugas de agua; permitiendo la refrigeración de los diferentes componentes como se ilustra en el Gráfico N.2. 


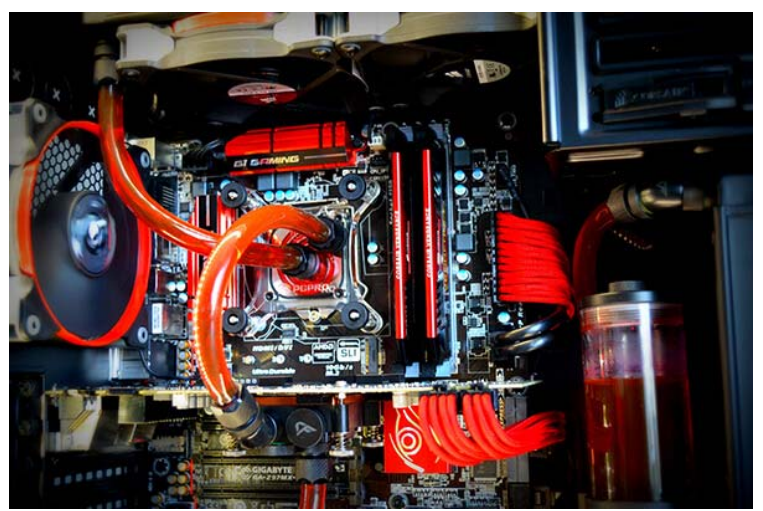

GRÁFICO N. 2:Refrigeración líquida Custom en un ordenador

(PCPRO, 2017).

Customestá conformado por los elementos siguientes:

$\checkmark$ Bomba:Esta destinada a impulsar el líquido por todos los componentes que se encuentran en el circuito.

$\checkmark$ Bloque:Su función es a realizar es intercambiar el calor que se genera y el líquido que se transporta en el componente a refrigerar.

$\checkmark$ Radiador:Elemento el cual cumple la función de enfriar el líquido caliente que viene de los distintos bloques.

$\checkmark$ Depósito:Elemento opcional pero para un mayor rendimiento es necesario,ya que facilita el llenado y limpiado del circuito.

$\checkmark$ Colector o Tubo:Elemento que hace que su función principal es hacer fluir el refrigerante por todos los elementos del ordenador en especial los circuitos.

$\checkmark$ Rácores: Une los tubos existen con los componentes del ensamblado.

$\checkmark$ Líquido refrigerante:Elemento que su función principal es pasar por cada uno de los componentes del ordenador y así bajar su temperatura.

$\checkmark$ Biocida:Liquido que se encarga de prevenir la aparición de algas en el líquido que recorre en el ordenador.(steemit beta, 2018).

\section{Refrigeración líquida Todo en Uno / All in one}

En este tipo de refrigeración cada una de sus piezas se adquiere individualmente, por lo cual se debe instalar la bomba y el radiador; existe mucha diferencia con los de custom por lo que no se necesita de un mantenimiento constante y su mayor ventaja se debe a que son fáciles de implementar; como se ilustra en el Gráfico N.3. 


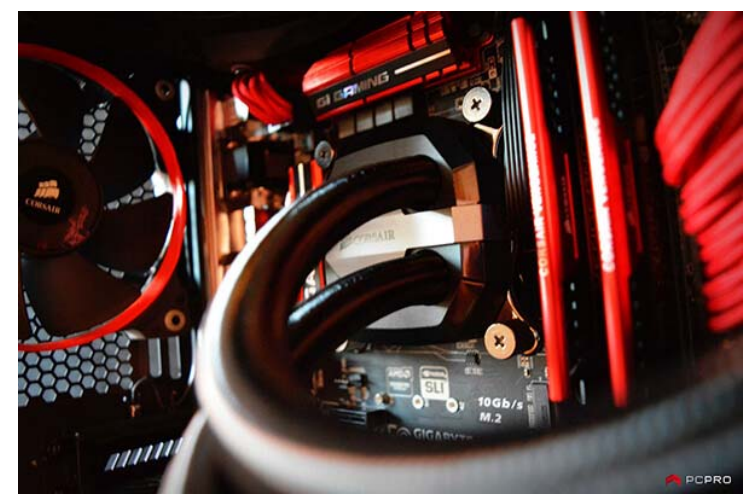

GRÁFICO N. 3:Refrigeración líquida “All in On”o “Todo en uno” en un ordenador.

(PCPRO, 2017).

\section{Funcionamiento}

El refrigerante se acumula en un depósito, es movido por una bomba, a través de los tubos que son los encargados para que circulen por los componentes; cuando el fluido ha cumplido su proceso, se dirige al radiador para enfriar el calor producido, y bombear nuevamente, formando así un ciclo cerrado de refrescamiento.

Se fundamenta en el principio de transferencia ya que la energía calórica va del objeto más caliente a un objeto más frío o más refrigerado.El proceso que realiza el refrigerante es en recorrer todos los circuitos del ordenador y este a su vez pasa por la pieza denominada radiador para así enfriarse y poder volver a realizar el mismo proceso al que está destinado.(Culturación, 2010).

Para que se realice una refrigeración correcta se debe tener en cuenta los requerimientos o normas que implica la adquisición e implementación de esta técnica.

\section{Refrigeración al ambiente externo}

Las condiciones de temperatura que debe tener si se encuentra en un ambiente externo se fundamentan en lo siguiente:

$\checkmark$ Clima del área donde se encuentre el ordenador.

$\checkmark$ La hermetización de la implementación de la refrigeración líquida.

$\checkmark$ Correcto mantenimiento a un ordenador. 
$\checkmark$ Eliminación de polvo y grasa (es recomendable que se tenga el ordenador con aire acondicionado).

$\checkmark$ Si la temperatura oscila entre los 10 a $32^{\circ}$, esto se hace para que no exista ningún inconveniente en el sistema de enfriamiento.

\section{Refrigeración al ambiente interno}

El ordenador debe tener las siguientes especificaciones en su temperatura y se fundamenta en las siguientes:

$\checkmark$ Dentro del ordenador debe quedar espacio para los tubos que se implementen no tengan un espacio reducido.

$\checkmark$ El CPU debe quedar completamente cerrado y atornillado debido a que el polvo o la suciedad puede ingresar.

$\checkmark$ Exposición al sol lo que puede afectar a nuestro ordenador.

$\checkmark$ Se debe configurar correctamente las conexiones, teniendo en cuento una mala conexión o un corto circuito puede dañar el equipo.

$\checkmark$ Mantener habilitadas las opciones de suspensión, hibernación y apagado del ordenador para que no se presente fallas en el sistema.(López, 2017).

\section{RESULTADOS}

Con la implementación de esta técnica en los ordenadores como lo es la refrigeración líquida se pretende que los usuarios conozcan las nuevas técnicas de enfriamiento y sus componentes; y adquieran conocimientos del cómo usarlas, así mismo de cómo se encuentra estructurado.

El impacto que se obtuvo en diferentes usuarios fue aceptable, e incluso los motivó a profundizar el tema conocer en que tecnologías se pueden implementar estas nuevas técnicas, así mismo sus beneficios como lo es un mayor rendimiento en un ordenador. 


\section{CONCLUSIONES}

Con el avanzar del tiempo las tecnologías se van innovando lo cual exige un mayor rendimiento en los ordenadores más de lo habitual y al implementar este sistema de refrigeración no solo aumentará el rendimiento del ordenador sino al mismo tiempo aumentará la durabilidad de los componentes que conforman el mismo.

La investigación resultó novedosa debido a que se pretendió que los usuarios adquirieran un mayor conocimiento de cómo implementar y utilizar esta técnica así mismo con el tiempo aportará nuevos conocimientos de las nuevas tecnologías de una manera aceptable.

\section{REFERENCIAS}

Culturación. (11 de Septiembre de 2010). Cuándo utilizar refrigeración líquida en tu computadora. Obtenido de culturacion.com: http://culturacion.com/cuando-utilizarrefrigeracion-liquida-en-tu-computadora/

Franco , R. (19 de Diciembre de 2013). Enfriamiento tradicional vs enfriamiento líquido: ¿cuál es mejor? Obtenido de hipertextual.com: https://hipertextual.com/archivo/2013/12/enfriamiento-aire-vs-liquido/

López, I. (30 de Noviembre de 2017). La refrigeración de las computadoras / CoolingComputers. Obtenido de jovenclub.cu: https://revista.jovenclub.cu/larefrigeracion-de-las-computadoras/

Morza. (19 de Febrero de 2018). Refrigeración líquida (informática). Obtenido de wikipedia.org:

https://es.m.wikipedia.org/wiki/Refrigeraci\%C3\%B3n_1\%C3\%ADquida_(inform\%C3 \%A1tica)

PCPRO. (16 de Diciembre de 2017). REFRIGERACIÓN LIQUIDA. Obtenido de pcpro.es: https://pcpro.es/componentes-pc-gaming/refrigeracionliquida/\#TIPOS_DE_REFRIGERACIONES_LIQUIDAS

Richards. (22 de Noviembre de 2016). culturacion.com. Obtenido de Cuándo utilizar refrigeración líquida en tu computadora: http://culturacion.com/cuando-utilizarrefrigeracion-liquida-en-tu-computadora/ 